\title{
3D printing applications for percutaneous structural interventions in congenital heart disease
}

\author{
Hannah Tredway ${ }^{1}$, Nikhil Pasumarti ${ }^{2}$, Matthew A. Crystal ${ }^{2}$, Kanwal M. Farooqi ${ }^{2}$ \\ 'Department of Pediatrics, New York Presbyterian/Columbia University Irving Medical Center, New York, NY 10032, USA. \\ ${ }^{2}$ Division of Pediatric Cardiology, Department of Pediatrics, New York Presbyterian/Columbia University Irving Medical Center,
} New York, NY 10032, USA.

Correspondence to: Dr. Kanwal M. Farooqi, New York Presbyterian/Columbia University Irving Medical Center, Morgan Stanley Children's Hospital, 3959 Broadway, CHN 2, New York, NY 10032, USA. E-mail: kf2549@cumc.columbia.edu How to cite this article: Tredway H, Pasumarti N, Crystal MA, Farooqi KM. 3D printing applications for percutaneous structural
interventions in congenital heart disease. Mini-invasive Surg 2020;4:78. http://dx.doi.org/10.20517/2574-1225.2020.77

Received: 31 Jul 2020 First Decision: 4 Sep 2020 Revised: 16 Sep 2020 Accepted: 27 Sep 2020 Published: 6 Nov 2020

Academic Editor: Bobak Mosadegh Copy Editor: Cai-Hong Wang Production Editor: Jing Yu

\begin{abstract}
The past several decades have seen remarkable advancements in percutaneous interventions for treatment of congenital heart disease (CHD). These advancements have been significantly aided by improvements in noninvasive diagnostic imaging. The use of three-dimensional (3D) printed models for planning and simulation of catheter-based procedures has been demonstrated for numerous cardiac defects and has been shown to reduce complications, procedure times, and limit radiation exposure. This paper reviews the process by which patient-specific 3D cardiac models are produced, as well as numerous applications of these models for use in percutaneous interventions in CHD.
\end{abstract}

Keywords: 3D models, pediatric interventional cardiology, congenital heart disease

\section{INTRODUCTION}

Over the past several decades, there has been a tremendous reduction in the morbidity and mortality associated with congenital heart disease (CHD) treatment. The wide array of anatomic pathologies can make diagnosis and management of these defects particularly challenging. Advances in the therapies for CHD have been aided largely by improvements in noninvasive diagnostic imaging. Traditional echocardiography demonstrates cardiac anatomy in two-dimensional (2D) planes, thereby limiting one's ability to fully visualize complex intracardiac structures and spatial relationships. While three-dimensional 
(3D) printing has been in use since the 1980s, recent application of this technique to the field of CHD has aided in both diagnosis of cardiac defects and pre-procedural planning, and has been shown to be particularly beneficial in guiding treatment of patients with more complex intracardiac anatomy ${ }^{[1-4]}$.

$3 \mathrm{D}$ reconstruction of imaging data from computed tomography (CT), magnetic resonance imaging (MRI), and $3 \mathrm{D}$ echocardiography allows for enhanced understanding of complex intracardiac anatomy prior to surgical or catheter-based procedures ${ }^{[5]} .3 \mathrm{D}$ models have been shown to be especially useful in devising percutaneous interventions for treatment of CHD. In recent years, there has been a remarkable evolution of catheterization techniques and technologies, including the development of numerous minimally invasive techniques to treat patients with $\mathrm{CHD}^{[3,6]}$. Catheter-based interventions have become the standard of care for a variety of procedures involving valve pathology, septal defects, and vascular abnormalities ${ }^{[6]} .3 \mathrm{D}$ models have the potential to provide additional anatomic insight and can be used to mimic device or stent implantations prior to the procedure. This is particularly useful for interventional cardiac procedures, as 3D models can be used to ascertain optimal shape and size of the device, understand how the device will fit into a specified location, and simulate the procedure in order to determine the optimal approach ${ }^{[1,2]}$. These models also enable proceduralists to perform entire procedures beforehand, thereby providing a means to anticipate complications, reduce radiation exposure, and potentially improve patient outcomes ${ }^{[5]}$. Here, we discuss the process by which patient-specific 3D cardiac models are produced, as well as numerous applications of these models for use in percutaneous interventions in CHD.

\section{IMAGE POSTPROCESSING}

Prior to creating a 3D model, a volumetric imaging dataset is acquired. CT and MRI are the most commonly used modalities for creating 3D reconstructions, although echocardiography and rotational angiography have also been used ${ }^{[5]}$. Preference of one modality over another depends largely on the experience of the center, the structure of interest, and age of patient. CT has been shown to be the easiest modality for model creation as it allows for particularly detailed segmentation of great vessels and intracardiac anatomy due to high spatial resolution ${ }^{[5]}$. Alternatively, MRI or contrast-enhanced magnetic resonance angiography offers whole heart 3D datasets while avoiding radiation exposure, which is preferred in younger patients ${ }^{[1]}$. More recently, there have been several reports on the use of $3 \mathrm{D}$ echocardiography in creating 3D reconstructions. Novel echocardiographic transducers as well as advancements in software and hardware have enhanced echocardiographic images, making them more suited for $3 \mathrm{D}$ modeling ${ }^{[7]}$. The use of echocardiography is beneficial as it is more widely available and avoids both radiation exposure and the necessity of radiocontrast administration ${ }^{[1]}$. This modality, however, has an inferior tissue-to-blood pool contrast, which makes image segmentation significantly more challenging. Echocardiography is also the preferred means by which to visualize cardiac valves and the atrial septum, which are poorly delineated in both CT and MRI. Hybrid imaging techniques have also been developed, which combine cross-sectional datasets with ultrasound in order to create complete heart models with embedded valve leaflets for more comprehensive visualization of intracardiac anatomy ${ }^{[2,7]}$.

Following the acquisition of the imaging dataset, a $3 \mathrm{D}$ rendering is created through a process known as segmentation ${ }^{[8]}$. The files are first uploaded as a DICOM (Digital Imaging and Communications in Medicine) dataset into 3D visualization software, such as Mimics (Materialise, Belgium), or open-source software, such as 3D Slicer (Slicer Wiki). Pixel-intensity-based thresholding is then employed to highlight the blood pool within the desired region. Subsequently, regions of interest are isolated through manual or semi-automatic techniques. The process of segmentation is the most time-consuming step of creating a $3 \mathrm{D}$ reconstruction. Accuracy of the model is largely determined by blood pool-to-tissue contrast, spatial resolution of the imaging technique, motion artifact of the image, and the technician's understanding of anatomic relationships ${ }^{[1,8]}$. The $3 \mathrm{D}$ rendering is then imported as a stereolithography (stl) file into a $3 \mathrm{D}$ visualization software, such as 3-Matic (Materialise, Belgium), or open source programs, such as Blender 
or Owlet, for post-processing to establish a print-compatible model ${ }^{[1,8]}$. This process involves converting the object into a meshed surface file, hollowing the model, smoothing surfaces, and trimming vessels or chamber walls in order to visualize the area of interest ${ }^{[5]}$. The $3 \mathrm{D}$ rendered object is converted into a computer-aided design format that can be converted into a physical object using a $3 \mathrm{D}$ printer ${ }^{[8]}$.

Once complete, the $3 \mathrm{D}$ rendering undergoes rapid prototyping on a $3 \mathrm{D}$ printer. Capabilities of $3 \mathrm{D}$ printers vary based on build volume, layer resolution, materials, and colors available ${ }^{[\rho]}$. The print technology utilized should be chosen based on the specific goal of the heart model. In making this decision, the material needed, level of detail, and turnaround time are all taken into consideration. Options for 3D printing include fused deposition modeling (FDM), Colorjet, Polyjet printing, and selective laser sintering ${ }^{[1]}$. In FDM, a thermoplastic filament is extruded in a specified pattern that immediately hardens. This process typically has a shorter turnaround time and comes at a significantly lower $\operatorname{cost}^{[8]}$. Colorjet printing is an additive manufacturing technology in which a core material is spread in thin layers and solidified by extrusion of a color binder. This technology allows for recreation of highly complex geometries in relatively short production times ${ }^{[10]}$. Polyjet printing, in contrast, allows for higher resolution printing of multiple materials in different colors, but it is much more costly and thus less often employed for routine modeling ${ }^{[7]}$. This technique enables the use of flexible, translucent materials, which are optimal for rehearsing surgical procedures as they can be cut, retracted, and sutured in order to effectively simulate procedures $^{[1]}$. Additionally, selective laser sintering utilizes a high-power laser to fuse metal or ceramic powder, resulting in a highly accurate model. This method, however, is often cost prohibitive in comparison to other techniques ${ }^{[5]}$. For each technique, the print material is sequentially layered, and the final model is encased in support material, which can be removed manually or by soaking in a solution ${ }^{[9]}$.

\section{CLINICAL APPLICATIONS}

The use of $3 \mathrm{D}$ printed models has been described widely for numerous percutaneous interventions for the treatment of CHD. One of the most well described interventions in which $3 \mathrm{D}$ models play a role is transcatheter valve implantation. These procedures represent the fastest growing area of innovation in the field of pediatric interventional cardiology, with numerous devices developed in the last decade ${ }^{[8]}$. Transcatheter valve replacements are beneficial because they enable proceduralists to correct valve regurgitation or stenosis without the need for repeat surgical interventions over the course of the patient's $\operatorname{life}^{[2,11]}$. Among these procedures, pulmonary valve replacement in repaired cases of Tetralogy of Fallot and aortic valve replacement for aortic stenosis or regurgitation are the most widely described. Poterucha et al. ${ }^{[12]}$ described a case of repaired tetralogy of Fallot in which the native right ventricular outflow tract (RVOT) was deemed to be unfavorable for percutaneous intervention. A 3D model of the RVOT [Figure 1] was then developed using 3D rotational angiography, which helped the interventionalist identify a landing zone for implantation of a Melody Valve (Medtronic, Fridley, Minnesota). A study by Shievano and colleagues showed that the use of $3 \mathrm{D}$ printed models allowed for more accurate selection of candidates for successful percutaneous pulmonary valve implantation (PPVI) than 3D MRI reconstructions alone ${ }^{[13]}$. Qian et al. ${ }^{[14]}$ demonstrated the use of $3 \mathrm{D}$ printed models of the left ventricular outflow tract (LVOT) and aortic root of patients with aortic stenosis. The models approximated the precise anatomy and flexibility of the LVOT, which had substantial tissue calcifications, and were used to test valve implantation prior to the procedure. This permitted the proceduralists to assess the feasibility of the intervention and predict paravalvular leak after transcatheter aortic valve replacement ${ }^{[9,14]}$. To test paravalvular leak, the models underwent analysis of strain distribution using a maximum bulge index, which aided in prediction of the degree of leakage following percutaneous valve implantation. This technique ultimately assisted in identifying ideal candidates for percutaneous rather than surgical intervention ${ }^{[14]}$. Along the same lines, Ripley et al. ${ }^{[15]}$ studied the use of $3 \mathrm{D}$ models to replicate patient-specific aortic root anatomy prior to transcatheter aortic valve replacement, and they found that the models provide insight into how the patient anatomy will interact with implanted medical devices. This enables interventionalists to predict potential challenges in device placement and complications during or following the procedure. 

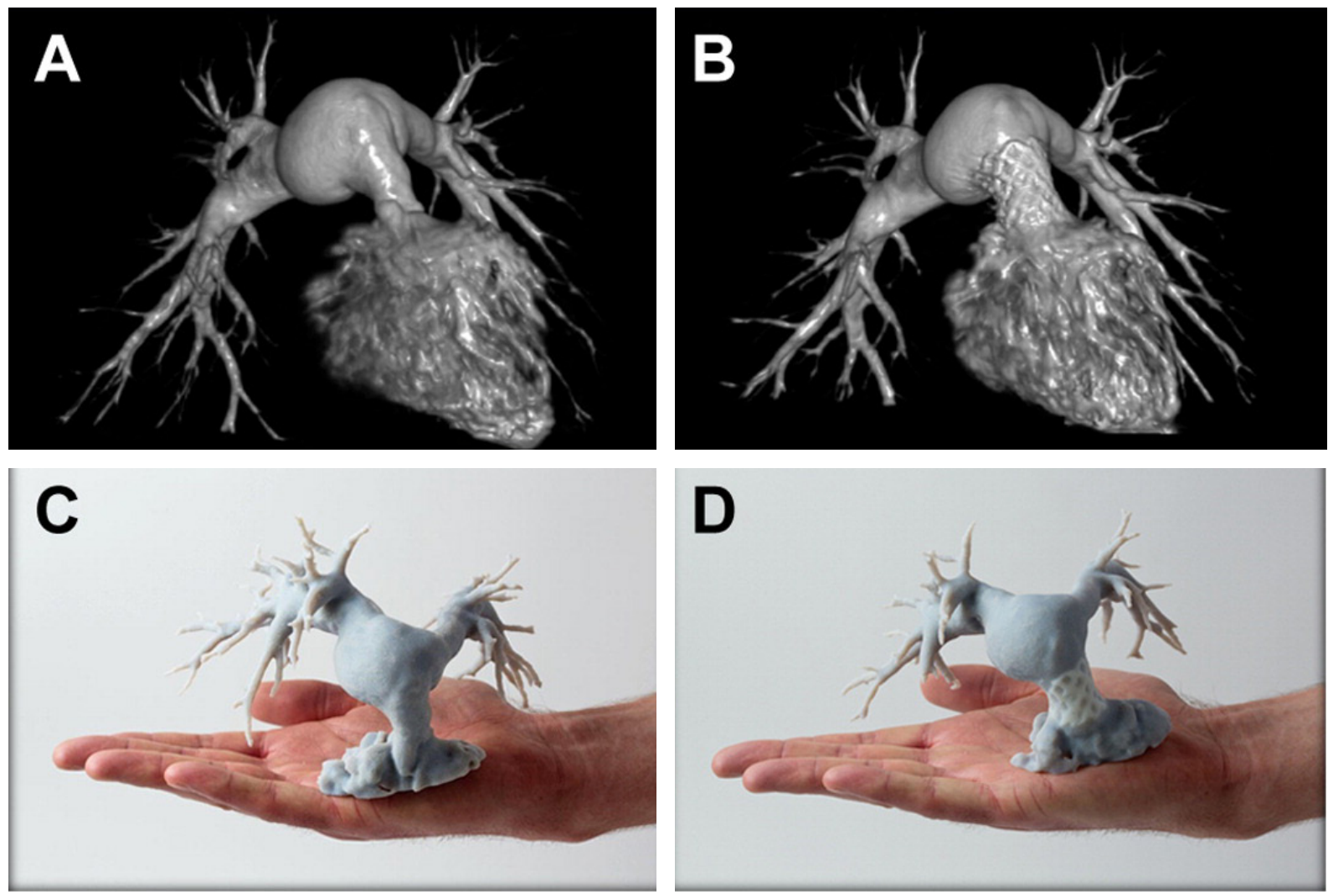

Figure 1. $3 D$ DynaCT reconstruction and $3 D$ printed models: pre-Melody valve implantation in the RVOT (A, C); and post-Melody valve implantation in the RVOT (B, D). Reprinted with permission from Poterucha et al. ${ }^{[12]}$. 3D: three-dimensional; RVOT: right ventricular outflow tract

An increasing number of devices have become available for atrioventricular valve repair using a percutaneous approach. Little et al. ${ }^{[16]}$ reported a case in which a $3 \mathrm{D}$ printed model was used to aid in procedural planning for a patient undergoing mitral valve repair with Mitraclip (Abbott, Abbott Park, Illinois). This group printed a multi-material $3 \mathrm{D}$ model in order to produce more realistic, deformable valve leaflets and recreate subvalvular calcium deposits within the adjacent myocardium. The model was then used to aid in the selection and sizing of the specific clip [Figure 2]. It enabled more accurate determination of a landing point for the device that avoided adjacent calcified tissue, and provided direct visualization of the effect of the implant on surrounding valve morphology and function ${ }^{[16]}$. Scanlan and colleagues performed a study in which patient-specific pediatric atrioventricular valves were modeled from $3 \mathrm{D}$ echocardiography ${ }^{[17]}$. The valves were printed and molded using custom software. The molded silicone valves were shown to be significantly more realistic for cutting and suturing, thus enhancing pre-procedural simulation. The technique is presently too time and labor intensive for widespread implementation ${ }^{[17]}$.

3D printed models are commonly used as guides for percutaneous closure of complicated atrial and ventricular septal defects. Velasco Forte et al. ${ }^{[18]}$ described a case in which a flexible, translucent 3D model was developed from a cardiac MRI in order to simulate the correction of a sinus venosus atrial septal defect (ASD). The model in this case allowed the interventionalists to accurately assess the anatomy and precise spatial relationships among the superior vena cava (SVC), left atrium (LA), and an anomalous pulmonary vein $(\mathrm{PV})$. The model was also used to determine the length of the stent required to close the defect and assess the positioning of the stent necessary to redirect blood flow from the anomalous PV to the LA without obstructing flow from other vessels [Figure 3]. Ultimately, a custom stent was successfully implanted into the SVC to close the sinus venosus ASD and commit the anomalous PV drainage correctly to the $\mathrm{LA}^{[18]}$. 


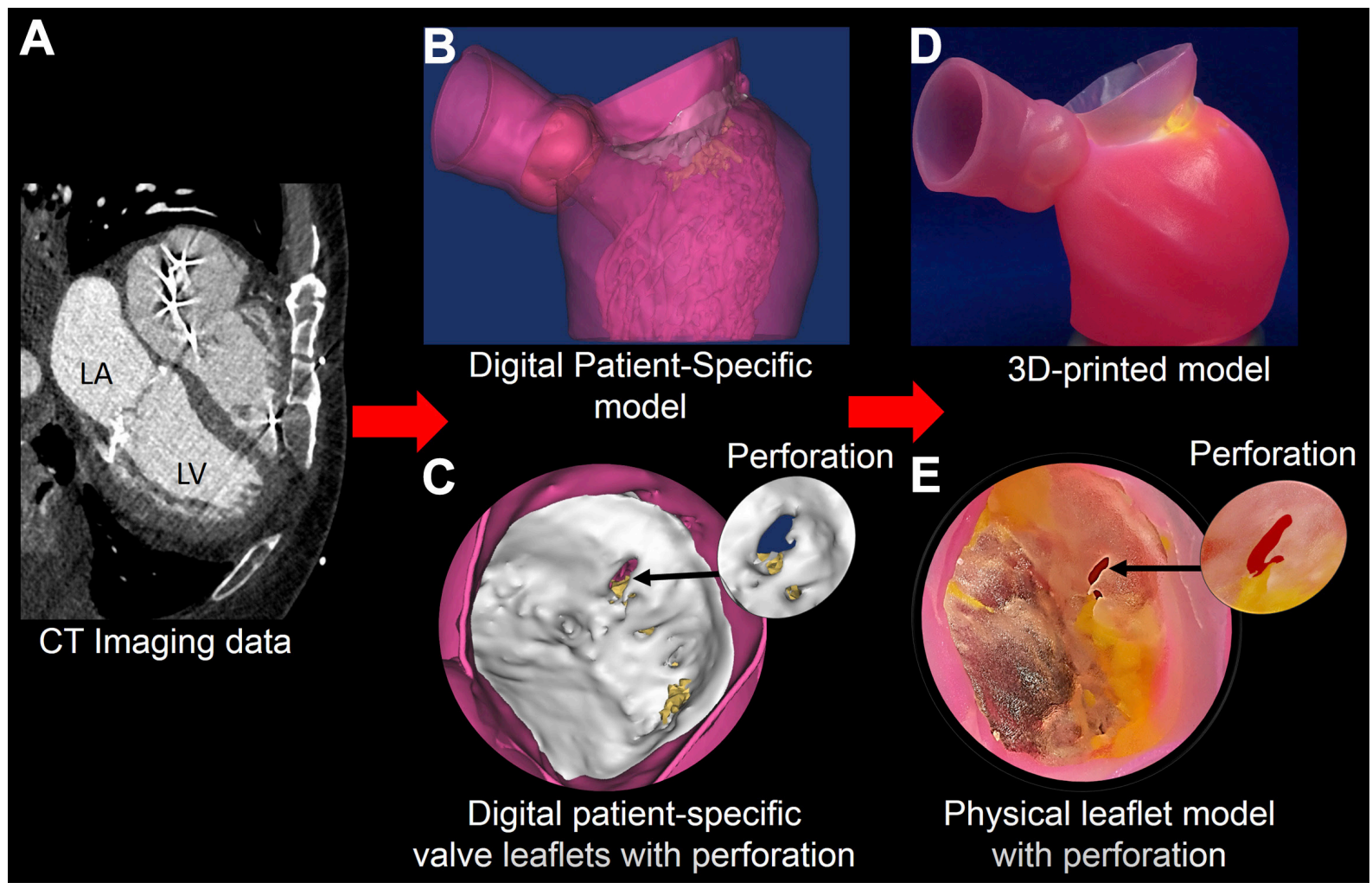

Figure 2. $C T$ images (A) are used to create a digital model (B) and to assign tissue properties (C); the multi-material patient-specific $3 D$ model $(D, E)$ is then printed to replicate the mitral valve leaflet geometry, regional calcium deposition, and pathology. Reprinted with permission from Little et al. ${ }^{[16]}$. CT: computed tomography; 3D: three-dimensional

A

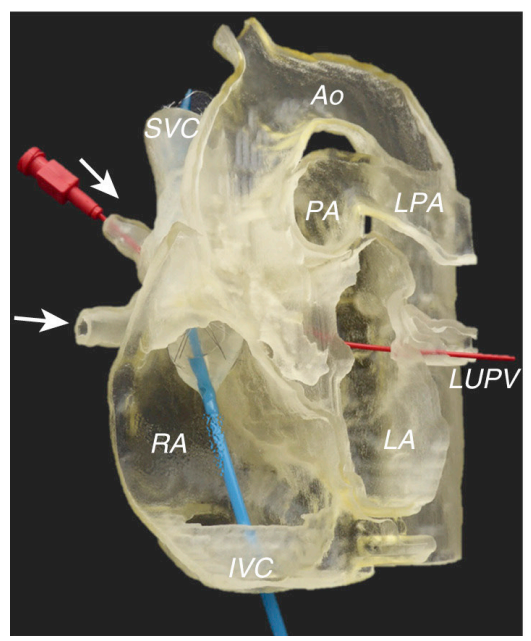

B

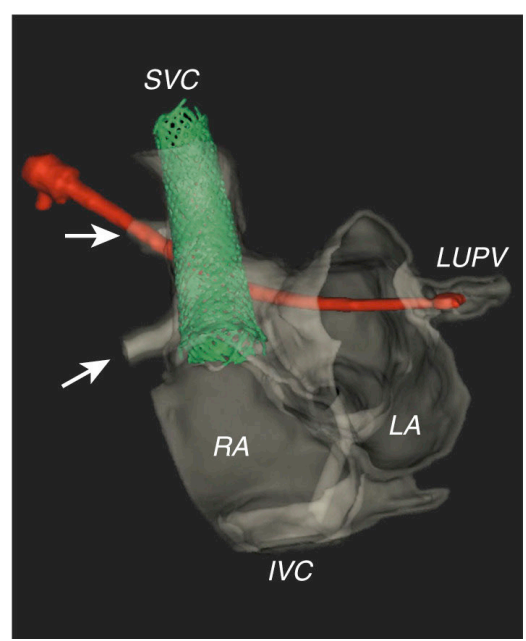

C

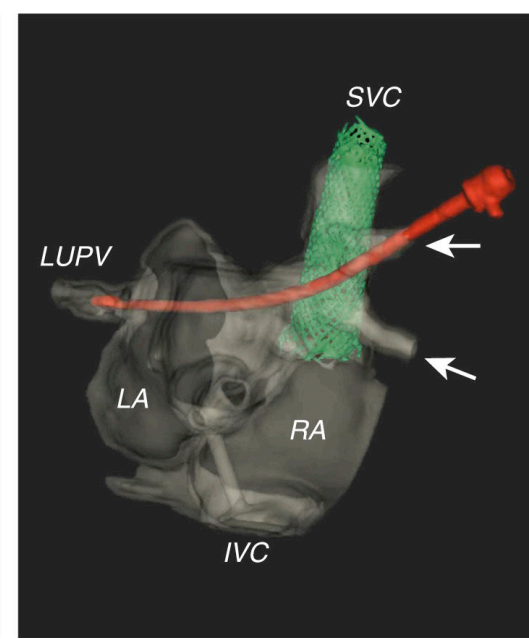

Figure 3. The flexible, translucent model (A) was examined to assess the relationship of the anomalous PVs (arrows) to the SVC and LA. A balloon-mounted stent catheter was placed in the SVC to RA junction (blue catheter), while a dilator (red) was passed from the anomalous right upper PV to the left upper PV. This model allowed for calculation of the length of the stent required to close the defect and redirect the flow of the partial anomalous pulmonary venous drainage toward the LA. CT of the model was performed using 3D rotational X-ray acquisition, shown from anteroposterior (B) and postero-anterior views (C) (dilator in red; stent in green). Reprinted with permission from Velasco Forte et al. ${ }^{[18]}$. PV: pulmonary vein; SVC: superior vena cava; LA: left atrium; RA: right atrium; CT: computed tomography; 3D: three-dimensional 
Another technically challenging percutaneous intervention that has the potential to be enhanced by patient-specific 3D printed models is endovascular stenting of the aorta in cases of aortic hypoplasia or coarctation. Placement of a stent within the aorta can result in many complications including stent migration, stroke, and occlusion of head and neck vessels by the stent itself. Valverde et al. ${ }^{[19]}$ presented a case in which a $3 \mathrm{D}$ model of a hypoplastic transverse aortic arch was created that closely mimicked the distensibility of the native vasculature and its response to stent delivery. In the case described, the endovascular stenting procedure was simulated on the printed model under fluoroscopic guidance prior to the percutaneous intervention. This simulation provided the proceduralists the opportunity to devise an optimal interventional approach, as well as determine the appropriate stent size, length, and position within the aorta ${ }^{[19]}$.

$3 \mathrm{D}$ printed models have recently been described for use in patients undergoing left atrial appendage (LAA) closure. Occlusion of the LAA in patients with atrial fibrillation significantly reduces thromboembolic risk in those who have contraindications to systemic anticoagulation ${ }^{[20]}$. Given the variable dimensions and morphology of the LAA, accurately sizing and positioning an occluder device in the orifice of the LAA can be challenging. Additionally, implanting a sub-optimally sized device to occlude the orifice can result in complications such as peri-device leakage, thrombus formation, device migration, and cardiac injury. Fan et al. ${ }^{[20]}$ conducted a study assessing the utility of 3D printed models created from 3D trans-esophageal echocardiography to aid in the selection of an appropriately sized device [Figure 4]. They found that device sizing based on 3D-printed models was associated with higher implantation success, shorter procedural times, and fewer complications. Iriart et al. ${ }^{[21]}$ described their technique of printing the entire left atrium and atrial septum in addition to the LAA in order to determine the optimal orientation for transseptal puncture during device placement. They also found that the models are invaluable in training physicians and fellows and augmenting communication with patients ${ }^{[21]}$.

Percutaneous closure of patent ductus arteriosus (PDA) is another intervention that has the potential to benefit from the use of 3D printed models. Particularly in adult cases, the PDA can be long, tortuous, and calcified, which makes catheter-based device placement challenging. Matsubara and colleagues presented a case in which patient-specific 3D printed models were created to detail the precise anatomy of the proximal aorta, aortic arch, PDA, and pulmonary artery ${ }^{[22]}$. These models allowed for selection of a particular device and exact size. They also allowed the interventionalists to simulate and practice device deployment within the models themselves, thereby decreasing fluoroscopic and procedural times ${ }^{[22]}$.

3D models can be instrumental in decision making to determine feasibility of transcatheter intervention. A recent case at our center involved a 78-year-old patient with a sinus venosus atrial septal defect with partial anomalous pulmonary venous return of the right upper pulmonary vein (RUPV) to the superior vena cava. A 3D model was created from a cardiac CT to demonstrate the relationship between the anomalous pulmonary venous return, atrial communication, and left atrium for potential use of a covered stent to reroute the RUPV flow. Although the cross-sectional imaging was helpful in delineating the pulmonary venous anatomy, the $3 \mathrm{D}$ model provided a much clearer picture of the spatial relationship among the RUPV, superior vena cava, and the left atrium. It was determined that use of a covered stent would result in occlusion of the RUPV in the position needed to ensure stent stability and avoid embolization. The patient will undergo surgical intervention for this congenital heart defect.

Finally, We described a case in which a large fistula, arising from the left coronary artery to the right atrium, was modeled in order to devise an approach for interventional closure [Figure 5A and B]. The $3 \mathrm{D}$ printed model enabled the interventionalists to consider several different approaches to transcatheter closure of the fistula [Figure 6]. Practicing the device closure on the 3D model demonstrated the feasibility of using a venous approach to access the fistula and provided insight on the optimal device to use for the procedure, with the goal of ultimately limiting procedure time and thereby reducing radiation exposure ${ }^{[23]}$. 

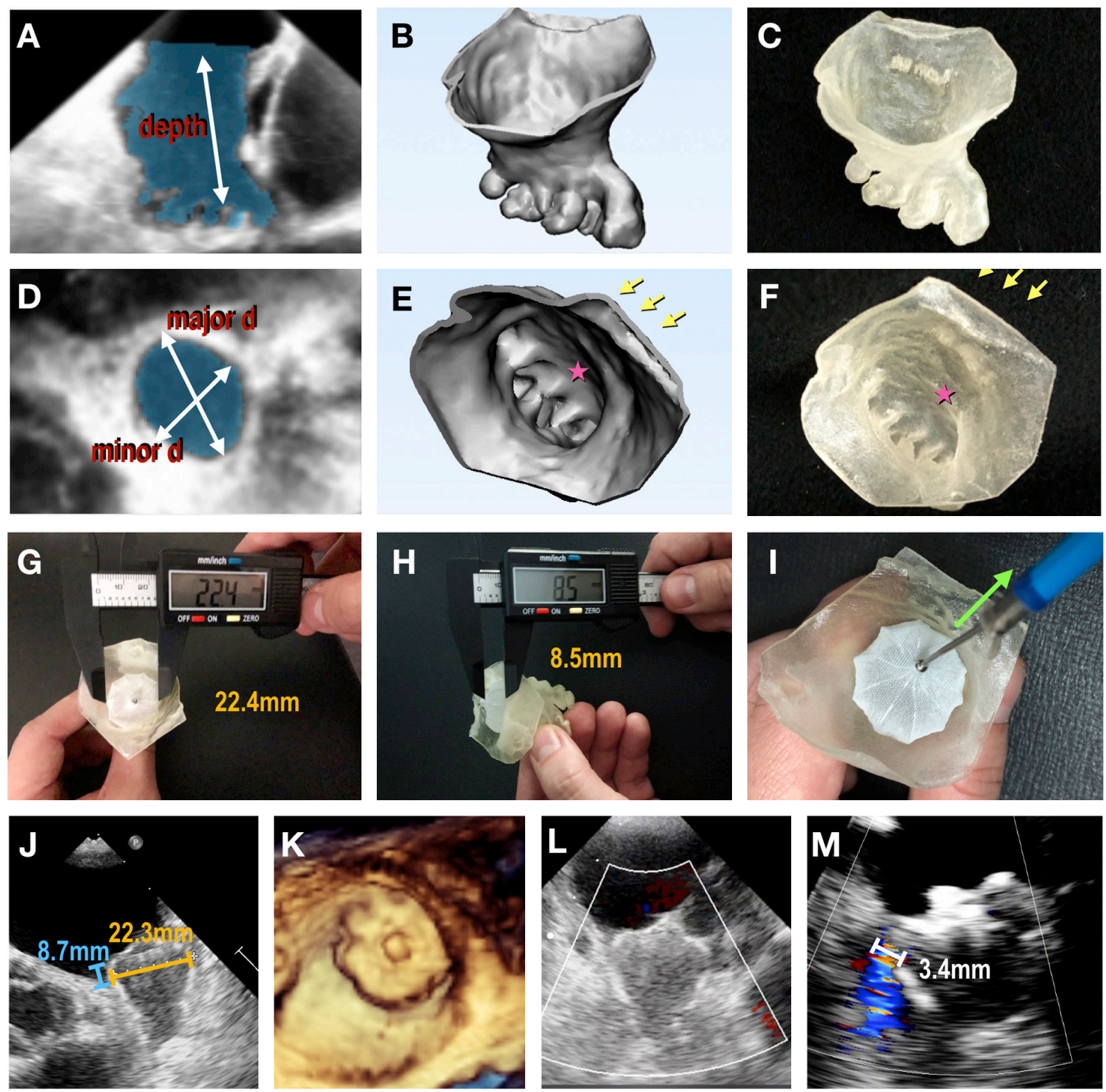

Figure 4. Echocardiography-based 3D printing of patient-specific models. Segmentation of LAA (shaded area) from 3D TEE data (A, D) is turned into a digital object (B, E), and printed using tissue-mimicking material (C, F). The major and minor ostial diameters and depth of the LAA are measured. Arrows denote pulmonary vein ridge; stars denote appendicular trabeculations. Closure devices are then sized and placed within the 3D model (G-I), and device compression and $(\mathrm{H})$ protrusion are measured using a digital caliper. Device stability is assessed using the tug-test (I). Device placement visualized on TEE $(\mathrm{J}-\mathrm{L})$, and color Doppler assessment showing no peri-device leak (M). Reprinted with permission from Fan et al. ${ }^{[20]}$. LAA: left atrial appendage; TEE: trans-esophageal echocardiogram; CT: computed tomography; 3D: three-dimensional

\section{LIMITATIONS}

There are numerous limitations to creating and using $3 \mathrm{D}$ printed models that have prevented widespread adoption in most programs ${ }^{[8]}$. A major consideration is that the creation of $3 \mathrm{D}$ models is a time-intensive process requiring familiarity with segmentation and computer automated design software, as well as an indepth understanding of cardiac morphology ${ }^{[5]}$. There is no standardized approach to creating these models, which can ultimately result in a wide variation in the quality of models produced ${ }^{[7]}$. This was evidenced by Burkhardt et al. ${ }^{[24]}$ in an article evaluating the inter-operator variability in modeling the RVOT based on the threshold chosen for the initial segmentation. Another limitation is that rigid, or even flexible, 3D 

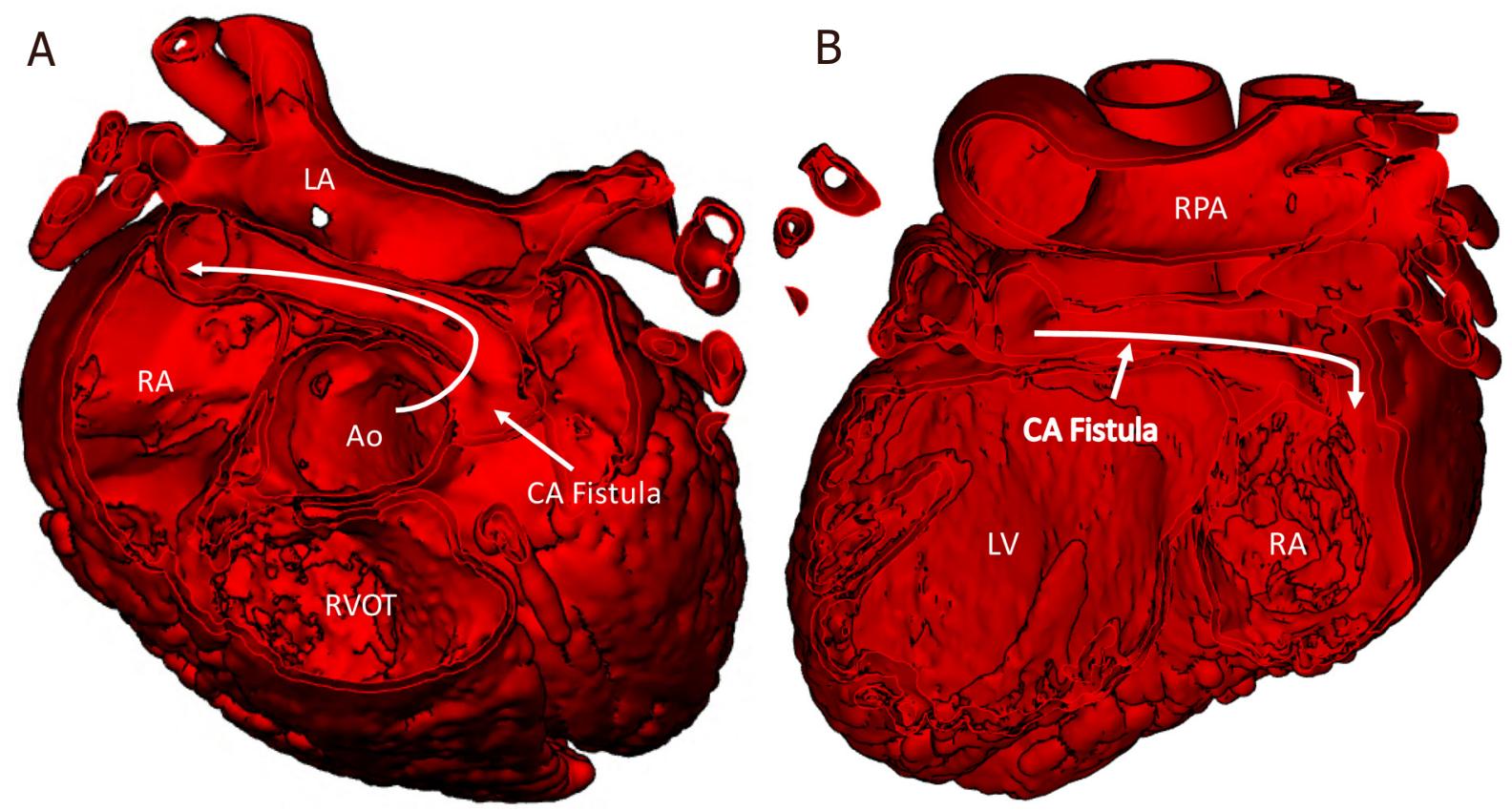

Figure 5. The course of the coronary fistula (CAF) is viewed from a short axis view of the heart. From the leftward aspect of the aortic root, it courses posterior to the aorta $(A o)$ and rightward to drain into the right atrium (RA) ( $A$ ); coronal view of the heart, as viewed from the posterior aspect, reveals the course of the CA fistula, almost parallel to the right pulmonary artery (RPA) from left to right to drain into the right atrium (B). CA: coronary artery; LA: left atrium; RVOT: right ventricular outflow tract

printed models only provide a snapshot of the cardiac structure at a specific point in the highly dynamic cardiac cycle, thus limiting our understanding of how these structures will change over the course of one heartbeat. Finally, creation of these models, while helpful in procedural planning, is not reimbursed by most insurance companies, and the prohibitively high cost of the software and $3 \mathrm{D}$ printing equipment significantly limits their utility on a routine basis ${ }^{[1]}$. More studies are required to further assess the costeffectiveness and diagnostic accuracy of these models prior to widespread implementation in the field of pediatric cardiology.

\section{FUTURE DIRECTIONS}

Recently, there has been movement toward developing materials that more closely mimic the feel and behavior of myocardium, valve leaflets, and vessel walls. Novel materials combined with the use of multiple imaging modalities could also aid in enhanced identification of valve tissue, chordae tendineae, and other structures that are less well defined with current methods. Developing models that accurately mimic both healthy and pathologic tissue would be invaluable in implementing these models routinely ${ }^{[15]}$. This advancement would not only allow for more accurate procedural planning but also be invaluable in training surgeons and interventionalists ${ }^{[2]}$. Further advances in imaging techniques and software to ease the burden of segmentation would allow for more widespread implementation of this technology to a broader range of conditions. Finally, 3D printing has the potential to aid in the design and construction of patientspecific catheter-based devices for numerous percutaneous interventions, which would further help to decrease procedural complications and improve long-term outcomes.

\section{CONCLUSION}

3D printed models have become increasingly invaluable tools in the field of pediatric cardiology. These models improve diagnostic ability, guide perioperative planning, and have thereby ushered in a vast array of new surgical and interventional approaches and techniques. Interventional cardiology has particularly 


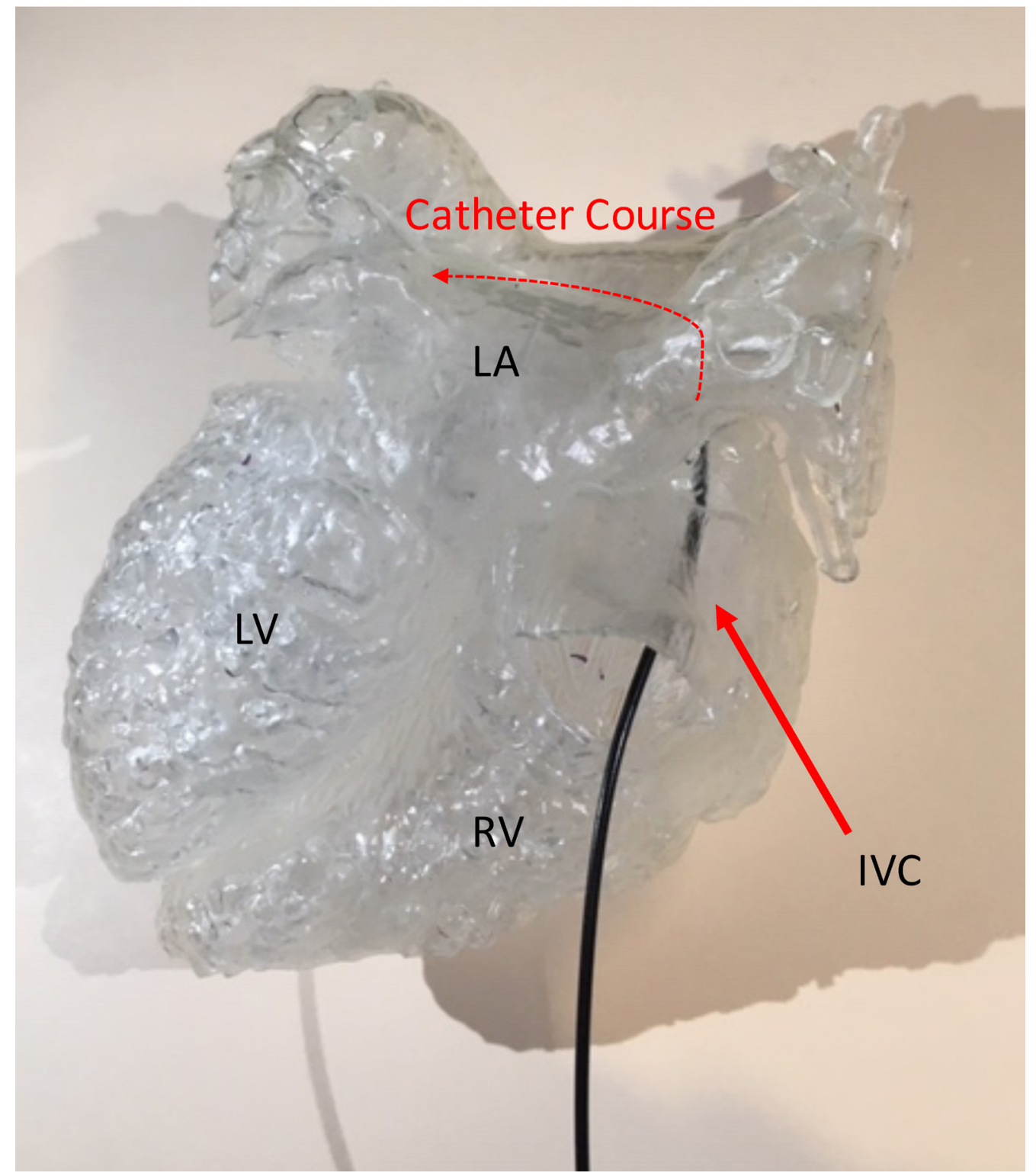

Figure 6. 3D printed model of the CA printed in a clear resin (Formlabs, Somerville, MA) allows planning of the transcatheter approach to closure of the fistula as viewed from the posterior aspect. A catheter courses from the inferior vena cava through the fistula (red dotted line). 3D: three-dimensional; CA: coronary artery; LA: left atrium; LV: left ventricular; RV: right ventricular; IVC: inferior vena cava

benefitted from the advancement of $3 \mathrm{D}$ modeling, as the models can be used to devise and adjust procedural approaches and practice percutaneous procedures, which has the potential to drastically reduce complications, decrease procedure times, and significantly limit radiation exposure. Ultimately, the use of $3 \mathrm{D}$ models has significantly improved our ability to practice personalized medicine and has helped to enhance the care of patients with cardiac defects through percutaneous procedures.

\section{DECLARATIONS}

\section{Authors' contributions}

Planning the manuscript content, writing individual sections, proofreading and approval of the final manuscript version: Tredway H, Pasumarti N, Crystal MA, and Farooqi KM 


\section{Availability of data and materials}

Not applicable.

\section{Financial support and sponsorship}

None.

\section{Conflicts of interest}

All authors declared that there are no conflicts of interest.

\section{Ethical approval and consent to participate}

Not applicable.

\section{Consent for publication}

Not applicable.

\section{Copyright}

(c) The Author(s) 2020.

\section{REFERENCES}

1. Moore RA, Riggs KW, Kourtidou S, et al. Three-dimensional printing and virtual surgery for congenital heart procedural planning. Birth Defects Res 2018;110:1082-90.

2. Zampi JD, Whiteside W. Innovative interventional catheterization techniques for congenital heart disease. Transl Pediatr 2018;7:104-19.

3. Cantinotti M, Valverde I, Kutty S. Three-dimensional printed models in congenital heart disease. Int J Card Imaging 2017;33:137-44.

4. Chepelev L, Wake N, Ryan J, et al. Radiological Society of North America (RSNA) 3D printing Special Interest Group (SIG): guidelines for medical 3D printing and appropriateness for clinical scenarios. 3D Print Med 2018;4:1-38.

5. Forte MNV, Hussain T, Roest A, et al. Living the heart in three dimensions: applications of 3D printing in CHD. Cardiol Young 2019;29:733-43.

6. Kang SL, Benson L. Recent advances in cardiac catheterization for congenital heart disease. F1000Res 2018;7:1-13.

7. Olivieri LJ, Krieger A, Loke YH, et al. Three-dimensional printing of intracardiac defects from three-dimensional echocardiographic images: feasibility and relative accuracy. J Am Soc Echocardiogr 2015;28:392-7.

8. Bramlet M, Olivieri L, Farooqi K, Ripley B, Coakley M. Impact of three-dimensional printing on the study and treatment of congenital heart disease. Circ Res 2017;120:904-7.

9. Farooqi KM, Cooper C, Chelliah A, et al. 3D printing and heart failure: the present and the future. JACC Heart Fail 2019;7:132-42.

10. ColorJet Printing. Available from https://www.3dsystems.com/on-demand-manufacturing/colorjet-printing. [Last accessed on 29 Sep 2020]

11. Armillotta A, Bonhoeffer P, Dubini G, et al. Use of rapid prototyping models in the planning of percutaneous pulmonary valved stent implantation. Proc Inst Mech Eng H 2007;221:407-16.

12. Poterucha JT, Foley TA, Taggart NW. Percutaneous pulmonary valve implantation in a native outflow tract: 3-Dimensional DynaCT rotational angiographic reconstruction and 3-dimensional printed model. JACC: Cardiovasc Interv 2014;7:e151-2.

13. Schievano S, Migliavacca F, Coats L, et al. Percutaneous pulmonary valve implantation based on rapid prototyping of right ventricular outflow tract and pulmonary trunk from MR data. Radiology 2007;242:490-7.

14. Qian Z, Wang K, Liu S, et al. Quantitative prediction of paravalvular leak in transcatheter aortic valve replacement based on tissuemimicking 3D Printing. JACC Cardiovasc Imaging 2017;10:719-31.

15. Ripley B, Kelil T, Cheezum MK, et al. 3D printing based on cardiac CT assists anatomic visualization prior to transcatheter aortic valve replacement. J Cardiovasc Comput Tomogr 2016;10:28-36.

16. Little SH, Vukicevic M, Avenatti E, Ramchandani M, Barker CM. 3D printed modeling for patient-specific mitral valve intervention repair with a clip and a plug. JACC Cardiovasc Interv 2016;9:973-5.

17. Scanlan AB, Nguyen AV, Ilina A, et al. Comparison of 3D echocardiogram-derived 3D printed valve models to molded models for simulated repair of pediatric atrioventricular valves. Pediatr Cardiol 2018;39:538-47.

18. Velasco Forte MN, Byrne N, Valverde I, et al. Interventional correction of sinus venosus atrial septal defect and partial anomalous pulmonary venous drainage: procedural planning using 3D printed models. JACC Cardiovasc Imaging 2018;11:275-8.

19. Valverde I, Gomez G, Coserria JF, et al. 3D printed models for planning endovascular stenting in transverse aortic arch hypoplasia. Catheter Cardiovasc Interv 2015;85:1006-12.

20. Fan Y, Yang F, Cheung GSH, et al. Device sizing guided by echocardiography-based three-dimensional printing is associated with superior outcome after percutaneous left atrial appendage occlusion. J Am Soc Echocardiogr 2019;32:708-19.

21. Iriart X, Ciobotaru V, Martin C, et al. Role of cardiac imaging and three-dimensional printing in percutaneous appendage closure. Arch 
Cardiovasc Dis 2018;111:411-20.

22. Matsubara D, Kataoka K, Takahashi H, Minami T, Yamagata T. A patient-specific hollow three-dimensional model for simulating percutaneous occlusion of patent ductus arteriosus: Its clinical usefulness. International Heart Journal 2019;60:100-7.

23. Tredway H, Pasumarti N, Crystal MA, Shah AM, Farooqi KM. How I used a model in clinical practice. In: Modeling Congenital Heart Disease: Engineering a Patient-specific Therapy. Springer Nature 2021; Forthcoming 2021.

24. Burkhardt BEU, Brown NK, Carberry JE, et al. Creating three dimensional models of the right ventricular outflow tract: infuence of contrast, sequence, operator, and threshold. Int Journal Cardiovasc Imaging 2019;35:2067-76. 